\title{
COHEN-MACAULAY RINGS AND IDEAL THEORY IN RINGS OF INVARIANTS OF ALGEBRAIC GROUPS(1)
}

\author{
BY \\ RONALD E. KUTZ
}

\begin{abstract}
Theorem. Let $R$ be a commutative Noetherian ring with identity. Let $M=$ $\left(c_{i j}\right)$ be an $s$ by symmetric matrix with entries in $R$. Let $I$ the be ideal of $t+1$ by $t+1$ minors of $M$. Suppose that the grade of $I$ is as large as possible, namely, $\operatorname{gr} I=g=$ $s(s+1) / 2-s t+t(t-1) / 2$. Then $I$ is a perfect ideal, so that $R / I$ is Cohen Macaulay if $R$ is.

Let $G$ be a linear algebraic group acting rationally on $R=K\left[x_{1}, \ldots, x_{n}\right]$. Hochster has conjectured that if $G$ is reductive, then $R^{G}$ is Cohen-Macaulay, where $R^{G}$ denotes the ring of invariants of the action of $G$. The above theorem provides a special case of this conjecture. For $G=O(t, K)$, the orthogonal group, and $K$ a field of characteristic zero, the above yields:

Corollary. For $R$ and $G$ as above, $R^{G}$ is Cohen-Macaulay for an appropriate action of $G$.

In order to obtain these results it was necessary to prove a more general form of the theorem stated above, which in turn yields a more general form of the corollary.
\end{abstract}

0. Introduction. Let $G$ be a linear algebraic group acting rationally on an $n$ dimensional $K$-vector space (see [2, p. 94]). We may consider $G$ as acting on the 1-forms of $R=K\left[x_{1}, \ldots, x_{n}\right]$; the action then extends uniquely to $R$. Hochster has conjectured that if $G$ is reductive, then the ring of invariants $R^{G}$ is CohenMacaulay [10]. In particular, the hypothesis holds if $G$ is one of the classical groups.

The conjecture is known in the following cases (see [10], [11]):

(i) $G=\mathrm{GL}(t)$ acts on $K^{(r+s) t}$ via: $X, Y$ are $r$ by $t$ and $t$ by $s$ matrices of indeterminates, respectively, and $A \in G$ acts by taking entries of $X$ to those of $X A^{-1}$ and entries of $Y$ to those of $A Y$.

(ii) $G=\mathrm{SL}(t)$ and acts on a $t$ by $r$ matrix of indeterminates $X$ by taking $X$ to $A X$ for all $A \in G$.

(iii) $G=\mathrm{GL}(1)^{m}$, the $m$-torus, and the representation is arbitrary.

(iv) Various representations of products of $\mathrm{SL}(t)$ with several copies of $\mathrm{GL}(m)$, $m$ varying.

(v) $G$ is finite and the representation is arbitrary.

This paper establishes the result for the case where $G=O(t)$, the orthogonal group, and the representation is as in (ii) above. In this case $R^{G}=K$ [entries of $\left.X^{t} X\right]=K\left[U_{i j}\right] / Q$, where $\left(U_{i j}\right)$ is a symmetric matrix of indeterminates

Received by the editors March 5, 1973.

AMS (MOS) subject classifications (1970). Primary 13C05, 13Cl0; Secondary 15A72.

Key words and phrases. Cohen-Macaulay rings, ideals generated by minors of matrices, perfectideals, rings of invariants, orthogonal group.

(1) This paper contains the results of the author's Ph.D. thesis, University of Minnesota, 1972. 
and $Q$ is the ideal generated by the $t+1$ by $t+1$ minors of $\left(U_{i j}\right)$ [16]. In order to obtain this result it is necessary to establish the conjecture for the more complex group of the product of $O(t)$ with a product of several copies of $\mathrm{GL}(m)$, $m$ varying.

1. The ideal-theoretic result. The conjecture for $G=O(t)$ follows quickly from the strictly ideal-theoretic result:

Theorem 1. Let $R$ be a commutative Noetherian ring with identity. Let $M=\left(c_{i j}\right)$ be an s by symmetric matrix with entries in $R$. Let $H=\left(s_{0}, \ldots, s_{m}\right)$ be a strictly increasing sequence of nonnegative integers such that $s_{0}=0, s_{m}=s$, and $m<s$. Let $n$ be an integer, $0 \leq n \leq s$. Let $I=I_{H, n}=I_{H, n}(M)$ be the ideal of $R$ generated by the $t+1$ by $t+1$ minors of the last $s_{t}$ columns of $M, 1 \leq t \leq m$, and $c_{1, s-n+1}, \ldots, c_{1, s}$. Let $h$ be the least integer such that $s_{h} \geq n$. Suppose that $n=s_{i}$ or $n=s_{i}+1$ for some $i, 0 \leq i \leq m$, and that the grade of $I$ is as large as possible, namely $g_{H, n}=s(s+1) / 2-s m+h+s_{1}+\cdots+s_{m-1}$. Then I is perfect; see [11].

Further, if $K$ is a Noetherian domain, $X=\left(x_{i j}\right)$ is a symmetric matrix of indeterminates over $K$ and $R=K[X]=K\left[x_{i j}\right]$, then $I_{H, n}$ is a perfect radical ideal of grade $g_{H, n}$ when $n=s_{i}$ or $n=s_{i}+1$. If $n=s_{i}$, then $I_{H, n}(X)$ is prime.

Corollary. When $H=(0,1,2, \ldots, t-1, s)$ and $n=0, I_{H, n}$ is the ideal of $t+1$ by $t+1$ minors of $M$, and the foregoing results hold.

Of course, the ideals of the corollary are those referred to in the last paragraph of $\S 0$. To prove those ideals perfect, however, it was necessary to prove the perfection of the entire class of ideals in Theorem 1.

The proof of Theorem 1 will proceed as follows: In $\$ 4$ we show $\sqrt{ } I_{H, n}$ is prime if $n=s_{i} . I_{H, n}$ is shown to be radical for $0 \leq n \leq s$ in $\$ 5$, and the primary decomposition of $I_{H, n}$ is obtained. In $\$ 6$ the grade of $I_{H, n}$ is computed, and $\$ 7$ completes the proof of Theorem 1.

2. The invariant theoretic result. As stated in $\S 0$, the original motivation for this paper was to show that the ring of invariants of an action of $G=O(t, K)$ was Cohen-Macaulay. Specifically, if $U$ is a $t$ by $n$ matrix of indeterminates and $G$ acts on $R=K[U]$ by taking $U$ onto $A U$ for $A \in G$, it is known classically that $R^{G}$ is generated by the entries of $X=U^{t} U$ and the ideal of relations on $X$ is generated by the $t+1$ by $t+1$ minors of $X$ and the symmetry conditions (see Weyl [16, $\$ 15$ and 17]). Hence, from the corollary to Theorem 1, we have

Proposition 2.1. With $R$ and $G$ as above, $R^{G}$ is Cohen-Macaulay.

Since in order to obtain the corollary of Theorem 1 it was necessary to prove the perfection of a much larger class of ideals, it is natural to hope that some of these may lead to rings of invariants. In fact, when $n=0, K[X] / I_{H, 0}(X)$ is the ring of invariants of a product of an orthogonal group and a product of general linear groups. This is made explicit and proofs are provided in \$8. This and 
Theorem 1 is all that is necessary to establish the desired result, as $K[X] / I_{H, 0}(X)$ is Cohen-Macaulay if and only if $I_{H, 0}(X)$ is perfect ([11, Proposition 19]).

3. Describing sets for ideals. Hochster-Eagon $[11, \S 6]$ established conventions and results for ideals of the form of $I_{H, n}(M)$; we use a modification of these results.

Let $N$ be the set of nonnegative integers, let $K \subset R$ be rings, let $X$ be an $r$ by $s$ matrix with entries in $R$ (we permit "blank" matrices $\varnothing$, one or both of whose dimensions are 0 ), and let $\left.X\right|_{i}$ be the matrix formed from the last (rightmost) $i$ columns of $X$. Then $\left.X\right|_{i}=\varnothing$ if $i=0$, while $\left.X\right|_{i}=X$ if $i \geq s$. If $Y$ is another matrix with $r$ columns, $\left.(Y X)\right|_{i}=Y\left(\left.X\right|_{i}\right)$. Let $I_{t}=I_{t}(X)$ be the ideal of $R$ generated by the $t+1$ by $t+1$ minors of $X . I_{t}=(0)$ if $t \geq \min \{r, s\}$.

If $h=(t, i) \in N \times N$, define $I_{h}(X)=I_{t}\left(\left.X\right|_{i}\right)$. If $H \subset N \times N$, let $I_{H}(X)$ $=\sum_{h \in H} I_{h}(X)$. We call $H$ a describing set of $I_{H}$. If $I_{H}=I_{H^{\prime}}$, we call $H$ and $H^{\prime}$ equivalent describing sets. It is shown in [11] that any describing set is equivalent to one of the form $H=\left\{\left(0, s_{0}\right),\left(1, s_{1}\right), \ldots,\left(m, s_{m}\right)\right\}$ where $s_{0}<s_{1}<\cdots<s_{m}$ $=s$, and this may be simplified to $H=\left(s_{0}, s_{1}, \ldots, s_{m}\right)$. Such a describing set will be called a standard description.

Let $I_{H, n}(X)=I_{H}(X)+\left(x_{1, s-n+1}, \ldots, x_{1, s}\right)$, where $H$ is any describing set and may be assumed standard. For many purposes, including showing that $I_{H, n}(X)$ is radical, prime, or perfect, we may also assume that $s_{0}=0$ and $n<s$. The above ideas are discussed in detail in [11].

The only difference in the above conditions and those in [11] is that we are using $I_{t}\left(\left.X\right|_{i}\right)$ to denote the ideal generated by the $t+1$ by $t+1$ minors of the rightmost $i$ columns of $X$, while [11] uses the leftmost $i$ columns. In the case of [11] the two conventions are isomorphic, and the numbering is easier working from the left. In our case the proofs fail when working from the left. We wish to show that when $X$ is a matrix of indeterminates the ideals of the form $I_{H, n}(X)$ are radical. Let $X=\left(x_{i j}\right)$ be a 2 by 2 symmetric matrix of indeterminates over $K[X]$, $K$ a field. Then $I_{1}(X)+\left(x_{11}\right)=\left(x_{11}, x_{12}^{2}\right)$, which is not radical, but $I_{1}(X)+\left(x_{12}\right)$ $=\left(x_{12}, x_{11} x_{22}\right)$, which is radical.

We partially order the pairs $(H, n)$ for $H$ a standard description as follows: Partially order the standard description via: If $H=\left(s_{0}, \ldots, s_{m}\right)$ and $H^{\prime}$ $=\left(s_{0}^{\prime}, \ldots, s_{k}^{\prime}\right)$, then $H \leq H^{\prime}$ if $m \leq k$ and $s_{i} \leq s_{i}{ }^{\prime}, 0 \leq i \leq m$. Then $(H, n)$ $\leq\left(H^{\prime}, n^{\prime}\right)$ if $H \leq H^{\prime}$ and $n \leq n^{\prime}$. Note that if $(H, n) \leq\left(H^{\prime}, n^{\prime}\right)$, the generating set of $I_{H, n}$ is a subset of the generating set of $I_{H^{\prime}, n^{\prime}}$.

4. $\sqrt{ } I_{H, n}$ is prime if $n=s_{h}$. In this section we will show that $\sqrt{ } I_{H, n}(X)$ is prime where $X$ is a symmetric matrix of indeterminates over $K$, a Noetherian domain, where $H$ is a standard description and $n=s_{h}$. We obtain the result by first proving the case where $n=0$.

Lemma 4.1. Let $X=\left(x_{i j}\right)$ be a symmetric $n$ by $n$ matrix with entries in $K$, a field, such that rank $X \leq t, t \leq n$. Let $C_{j}$ denote the jth column of $X$, and let $j_{1}, \ldots, j_{t}$ 
be such that $C_{j_{1}}, \ldots, C_{j_{t}}$ span the column space of $X$. Let $A$ be the $n$ by $t$ matrix formed by $C_{j_{1}}, \ldots, C_{j_{i}}$. Under these conditions there exists $B$, a $t$ by $n$ matrix, such that $X=A B$. Then there exists a $t$ by $t$ symmetric matrix $S$ such that $A=B^{t} S$, so that $X=B^{t} S B$.

Proof. Let $N=\{1, \ldots, n\}, I=\left\{j_{1}, \ldots, j_{t}\right\}$. Then $A=\left.X\right|_{N \times I}$. Let $S=\left.X\right|_{I \times I}$. Clearly, $S$ is symmetric. We will show that $A=B^{t} S$. $B^{t} S=B^{t} S^{t}=(S B)^{t}$ $=\left(\left.X\right|_{I \times I} B\right)^{t}=\left(\left.A\right|_{I \times I} B\right)^{t}=\left(\left.A B\right|_{I \times N}\right)^{t}=\left(\left.X\right|_{I \times N}\right)^{t}=\left.X^{t}\right|_{N \times I}=\left.X\right|_{N \times I}$ $=A$. Q.E.D.

A generic point for a prime ideal $P$ of $R$ is a homomorphism from $R$ to a domain whose kernel is $P$. Hence to construct such a homomorphism is to prove the kernel is prime; this is the procedure used on the ideals in question.

Let $K \subset R$ be rings. If $X$ and $X^{\prime}$ are $r$ by $s$ and $r$ by $s^{\prime}$ matrices respectively over $R$, let $X * X^{\prime}$ be the $r$ by $s+s^{\prime}$ matrix obtained by juxtaposing $X$ and $X^{\prime}$. Left multiplication by an $r^{\prime}$ by $r$ matrix $Y$ distributes over $*$, that is, $Y\left(X * X^{\prime}\right)$ $=Y X * Y X^{\prime}$. Note that $K\left[X * X^{\prime}\right]=K\left[X, X^{\prime}\right]$.

For the rest of this section let $K$ be a Noetherian domain, let $X$ be an $s$ by $s$ symmetric matrix of indeterminates over $K$. For $\S 8$ it will be expedient to consider nonstandard descriptions in Proposition 4.2. Let $H=\left\{\left(t_{0}, i_{0}\right), \ldots\right.$, $\left.\left(t_{m}, i_{m}\right)\right\}$, where $t_{0}<\cdots<t_{m}$ and $i_{0}<\cdots<i_{m}$. Suppose $i_{m}=s$ and $t_{m}<s$. Let $I_{H, n}(X)$ be the ideal of $K[X]$ defined in $\S 3$. Denote $I_{H, 0}(X)=I_{H}(X)=I_{H}$; let $J_{H, n}(X)=\sqrt{ } I_{H, n}(X)$.

Proposition 4.2. $J_{H}(X)$ is prime.

Proof. Let $j_{k}=i_{k}-i_{k-1}, 0 \leq k \leq m$; let $U_{k}$ be a $t_{k+1}$ by $t_{k}$ matrix of indeterminates, $0 \leq k \leq m-1$; let $V_{k}$ be a $t_{k}$ by $j_{k}$ matrix of indeterminates, $0 \leq k \leq m$; let $S$ be a symmetric $t_{m}$ by $t_{m}$ matrix of indeterminates; let $Z=V_{m} *\left(U_{m-1} V_{m-1}\right) * \cdots *\left(U_{m-1} \cdots U_{k} V_{k}\right) * \cdots *\left(U_{m-1} \cdots U_{0} V_{0}\right)$; and let $W$ $=Z^{t} S Z$. Note that $U_{m-1} \cdots U_{k}$ is $t_{m}$ by $t_{k}$ so that $U_{m-1} \cdots U_{k} V_{k}$ is $t_{m}$ by $j_{k}$. Then $Z$ is $t_{m}$ by $s$ and $W$ is $s$ by $s$. Also, $W^{t}=\left(Z^{t} S Z\right)^{t}=Z^{t} S^{t} Z=Z^{t} S Z=W$, so $W$ is symmetric.

Consider the ring $K[W] \subset K\left[U_{k}, V_{k}, S\right]$. Let $\phi: K[X] \rightarrow K[W]$ be the mapping that takes entries of $X$ to corresponding entries of $W$ (or, briefly, takes $X$ to $W$ ), extended to a homomorphism. Since $K[W]$ is a domain, Proposition 4.2 will be proved if we show $J_{H}(X)=\operatorname{ker} \phi$.

To show $J_{H} \subset \operatorname{ker} \phi$, it is sufficient to show $I_{H} \subset \operatorname{ker} \phi$, i.e. we need to verify that for $h=(t, i)=\left(t_{k}, i_{k}\right) \in H, I_{h}(W)=(0)$. But if $Z_{j}=\left(U_{m-1} \cdots U_{j} V_{j}\right)$ $* \cdots *\left(U_{m-1} \cdots U_{0} V_{0}\right)$ so that

$$
\left.W\right|_{i}=Z^{t} S Z_{i}=Z^{t} S\left(U_{m-1} \cdots U_{i}\right)\left(V_{i} * \cdots * U_{i-1} \cdots U_{0} V_{0}\right),
$$

then $I_{t}\left(\left.W\right|_{i}\right)=I_{t}\left(Z^{t} S Z_{i}\right)=(0)$, since the factor $\left(V_{i} * \cdots * U_{i-1} \cdots U_{0} V_{0}\right)$ of $Z^{t} S Z_{i}$ has $t$ rows. 
To show ker $\phi \subset J_{H}(X)$, let $P$ be any prime of $K[X]$ such that $I_{H}(X) \subset P$. Let $X^{-}=X(\bmod P), D=K[X] / P=K\left[X^{-}\right]$, and let $L$ be the fraction field of $D$. By the definition of $I_{H}(X), X^{-}$is symmetric and rank $\left.X^{-}\right|_{i} \leq t$ for each $(t, i)=\left(t_{k}, i_{k}\right) \in H$. It is sufficient to show that under these conditions $X^{-}$can be "factored" over $L$ into the same form as $W$. For then the diagram

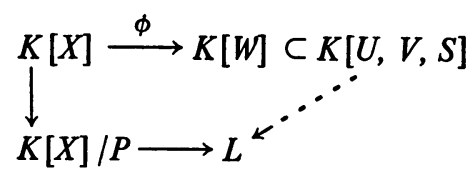

can be completed, showing that ker $\phi \subset P$.

Write $X^{-}=Y_{m} * \cdots * Y_{0}$, where $Y_{k}$ has $j_{k}$ columns. For each $k, 0 \leq k \leq m$, the rank of $Y_{k} * \cdots * Y_{0}$ is $\leq t_{k}$, so we can choose an $s$ by $t_{k}$ matrix $T_{k}$ over $L$ such that the column space of $T_{k}$ equals that of $Y_{k} * \cdots * Y_{0}$, choosing $T_{m}$ so that its columns are among those of $X^{-}$.

Since the column space of $T_{m}$ is a subspace of the column space of $T_{m+1}$ for each $k, 0 \leq k \leq m-1$, we can choose $U_{k}^{\prime}, t_{k+1}$ by $t_{k}$, so that $T_{k}=T_{k+1} U_{k}^{\prime}$. Let $U_{m}^{\prime}=T_{m}$. Since the column space of $Y_{k}$ is a subspace of the column space of $T_{k}$, we can choose $V_{k}^{\prime}, t_{k}$ by $j_{k}$, so that $Y_{k}=T_{k} V_{k}^{\prime}, 0 \leq k \leq m$.

Then, for $0 \leq k \leq m, T_{k}=T_{k+1} U_{k}^{\prime}=T_{k+2} U_{k+1}^{\prime} U_{k}^{\prime}=\cdots=U_{m}^{\prime} \cdots U_{k}^{\prime}$, and $Y_{k}=T_{k} V_{k}^{\prime}=U_{m}^{\prime} \cdots U_{k}^{\prime} V_{k}^{\prime}$, so that

$$
\begin{aligned}
X^{-} & =Y_{m} * \cdots * Y_{0}=\left(U_{m}^{\prime} V_{m}^{\prime}\right) * \cdots *\left(U_{m}^{\prime} \cdots U_{k}^{\prime} V_{k}^{\prime}\right) * \cdots *\left(U_{m}^{\prime} \cdots U_{0}^{\prime} V_{0}^{\prime}\right) \\
& =U_{m}^{\prime}\left[V_{m}^{\prime} *\left(U_{m-1}^{\prime} V_{m-1}^{\prime}\right) * \cdots *\left(U_{m-1}^{\prime} \cdots U_{0}^{\prime} V_{0}^{\prime}\right)\right]=U_{m}^{\prime} Z^{\prime} .
\end{aligned}
$$

But $U_{m}^{\prime}$ has as columns a spanning set of $X^{-}$, and $X^{-}=U_{m}^{\prime} Z^{\prime}$, so by Lemma 4.1, $U_{m}^{\prime}=\left(Z^{\prime}\right)^{t} S^{\prime}$, where $S^{\prime}$ is $t_{m}$ by $t_{m}$ symmetric. Hence $X^{-}=\left(Z^{\prime}\right)^{t} S^{\prime} Z^{\prime}$, as required.

To show $J_{H, n}(X)$ is prime for $n=s_{h}$, we need some preliminary results.

Proposition 4.3. Let $Y=\left(y_{1}, \ldots, y_{r}\right)$ and let $S=\left(s_{i j}\right)$ be an $r$ by $r$ symmetric matrix, $y_{i}, s_{i j}$ indeterminates. Then the entries of $Y S$ form an $R$-sequence in $K[Y, S]$.

Proof. Let $(Y S)$ denote the ideal generated by the entries of $Y S$. Since $(Y S)$ is homogeneous, we need only show $(Y S)$ has grade $r$, which will follow if $\sqrt{ }(Y S)$ has grade $r$. We will prove $\sqrt{ }(Y S)=\left(y_{1}, \ldots, y_{r}\right) \cap \sqrt{ }(\operatorname{det} S,(Y S))$, where $\operatorname{gr}\left(y_{1}, \ldots, y_{r}\right)=r$ and $\sqrt{ }(\operatorname{det} S,(Y S))$ is of grade at least $r$. The equality of the ideals is proved in Proposition 4.4; gr $\sqrt{ }(\operatorname{det} S,(Y S)) \geq r$ is proved in Proposition 4.5. That $\operatorname{gr}\left(y_{1}, \ldots, y_{r}\right)=r$ is obvious.

Proposition 4.4. $\sqrt{ }(Y S)=\left(y_{1}, \ldots, y_{r}\right) \cap \sqrt{ }(\operatorname{det} S,(Y S))$.

Proof. The left-hand side of the equality is clearly contained in the right-hand side. To show the opposite containment, it is sufficient to show $\left(y_{1}, \ldots, y_{r}\right)$ 
-(det $S,(Y S)) \subset(Y S)$, which follows if $y_{i}$ det $S \in(Y S)$. Let $Y S=\left(f_{1}, \ldots, f_{r}\right)$. Then

$$
\begin{aligned}
s_{11} y_{1}+\cdots+s_{1 r} y_{r}= & f_{1} \\
\vdots & \vdots \quad \vdots \\
s_{r 1} y_{1}+\cdots+s_{r r} y_{r} & =f_{r} .
\end{aligned}
$$

In $K(Y, S)$, since det $S \neq 0$, Cramer's rule yields

$$
y_{i} \operatorname{det} S=\left|\begin{array}{ccccc}
s_{11} & \cdots & f_{1} & \cdots & s_{1 r} \\
\vdots & & \vdots & & \vdots \\
s_{r 1} & \cdots & f_{r} & \cdots & s_{r r}
\end{array}\right| \text {. }
$$

Hence, in $K[Y, S]$, we may expand the right-hand side in terms of the $i$ th column, obtaining $y_{i}$ det $S=A_{1} f_{1}+\cdots+A_{r} f_{r} \in(Y S)$, as required.

Proposition 4.5. gr $\sqrt{ }(\operatorname{det} S,(Y S)) \geq r$.

Proof. Let $I=(\operatorname{det} S,(Y S)) \cdot \sqrt{ } I=\cap P_{\alpha}$ over primes $P_{\alpha}$ which contain $I$. Since gr $I=\mathrm{gr} \sqrt{ } I=\min \{\mathrm{gr} P \mid P$ is a minimal prime of $I\}$, we need show that if $P$ is a prime containing $I$, then $\mathrm{gr} P \geq r$. For each such $P$ we construct a ring $A(P)$ and an onto homomorphism $\phi: R=K[Y, S] \rightarrow A(P)$ such that $I \subset \operatorname{ker} \phi$ and

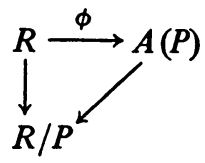

commutes. We then have ker $\phi \subset P$, so gr $P \geq \operatorname{gr} \operatorname{ker} \phi=\operatorname{dim} R-\operatorname{dim} A(P)$, which we can then compute.

Fix $P$, a prime containing $I$ in $R$. Let $Y^{-}, S^{-}$denote the images of $Y, S$, respectively in $R / P$. Since $\operatorname{det} S^{-}=0$, rank $S^{-}=t<r$. Further, $Y^{-} S^{-}$ $=(0, \ldots, 0)$ and $S^{-}$is symmetric. Let $L$ be the fraction field of $R / P$. Suppose the $j_{1}, \ldots, j_{t}$ th columns of $S^{-}$are a basis for the column space of $S^{-}$, and the $i_{1}, \ldots, i_{t}$ th rows of $S^{-}$are such that $\operatorname{det}\left(s_{i_{j_{m}}}\right) \neq 0, k, m=1, \ldots, t$.

We now construct $A(P)$. Let $U$ be an $r$ by $r$ symmetric matrix constructed as follows: Let the $j_{1}$ st column consist of $r$ indeterminates $u_{-, j_{j}}$. Symmetry determines one entry of the $j_{2}$ nd column; let the other entries consist of indeterminates. Proceed, filling in the $j_{k}$ th column with $r-k+1$ indeterminates $u_{-, j_{k}}$. The $k-1$ other entries will have been determined by the symmetry of $U$. Fill in the $j_{1}, \ldots, j_{t}$ th rows by symmetry, and the rest of $U$ so that $U$ has rank $t$. Working in $K\left(u_{i j}\right)$, the other entries are determined uniquely. Since every column is a linear combination of the $j_{i}, \ldots, j_{t}$ th columns, and $t$ entries are already fixed, the coefficients of the linear combinations are determined since the columns are a basis. Note that we have $(2 r-t+1) t / 2$ indeterminates. 
Let $V$ be the $t$ by $t$ matrix formed by restricting $U$ to its $j_{1}, \ldots, j_{t}$ th columns and $i_{1}, \ldots, i_{t}$ th rows. Let $V_{i, i_{n}}$ denote $V$ with its $i_{n}$ th row replaced by the corresponding entries of the $i$ th row of $U$. For $i \neq i_{1}, \ldots, i_{t}$, let $Y_{i}$ be the 1 by $r$ matrix whose $i$ th entry is det $V$, and whose other entries are zero. Let $\left\{k_{1}, \ldots, k_{r-t}\right\}=\{1, \ldots, r\}-\left\{i_{1}, \ldots, i_{t}\right\}$, where $k_{1}<\cdots<k_{r-t}$. Let $Z=z_{1} Y_{k_{1}}$ $+\cdots+z_{r-t} Y_{k_{r-1}}$, a 1 by $r$ matrix, where $z_{1}, \ldots, z_{r-t}$ are indeterminates. What we have accomplished is less complex than it appears. We have simply constructed the most general vector $Z$ that will be orthogonal to the column space of $U$, by setting $Y U=0$ (where $Y=\left(y_{1}, \ldots, y_{r}\right)$ ) and solving for $y_{n}, \ldots, y_{r_{t}}$ by Cramer's rule. The dimension of the solution space will be $r-t$, accounting for the $r-t$ "arbitrary multiples" $z_{1}, \ldots, z_{r-t}$.

We now have $A(P)$, namely $K[Z, U] \subset K\left(u_{i j}, z_{i}\right)$. Let $\phi: K[Y, S] \rightarrow K[Z, U]$ by taking $Y$ to $Z$ and $S$ to $U . I \subset$ ker $\phi$ is clear, since rank $U=t<r$ and $Z U=0$ by the construction of $Z$. To complete the argument, we need to show that in $L$, $Y^{-}$and $S^{-}$"factor" in the same form as $Z$ and $U$.

But this is clear, since the $j_{1}, \ldots, j_{t}$ th columns of $S^{-}$are a basis for the column space of $S^{-}$over $L$, the other columns are each a unique linear combination of the basis columns, where the linear combination is consistent with the symmetry, i.e. consistent with the method of formation of $U$. That $Y^{-}$"factors" in the form of $Z$ follows because, just as with $Z$, the condition $Y^{-} S^{-}=0$ and the restrictions on $S^{-}$enable us to use Cramer's rule to express $Y^{-}$as a linear combination of vectors of the form $Y_{\boldsymbol{k}_{i}}$.

Now gr ker $\phi=\operatorname{dim} R-\operatorname{dim} K[U, Z]=\operatorname{tr} \operatorname{deg}_{K} K(Y, S)-\operatorname{tr} \operatorname{deg}_{K} K(U, Z)$. In each case, this is given by the number of indeterminates over $K$, so $\operatorname{tr} \operatorname{deg}_{K} K(Y, S)=$ $r+r(r+1) / 2$, and $\operatorname{tr} \operatorname{deg}_{K} K(U, Z)=(2 r-t+1) t / 2+(r-t)$. Hence gr $P=\operatorname{gr} \operatorname{ker} \phi=$ $r+r(r+1) / 2-(2 r-t+1) t / 2-(r-t)=r+\left(r^{2}+r-2 r t+t^{2}-t-2 r+2 t\right) / 2=$ $r+\left[(r-t)^{2}-(r-t)\right] \geqslant r$, since $r \geqslant t$. Q.E.D.

Hence the entries of $Y S$ form an $R$-sequence. We use this and [11] to show that $J_{H, n}(X)$ is prime if $n=s_{h}$.

Proposition 4.6. Let $K$ be a Noetherian domain. Let $r=t_{m+1}>t_{m}>\cdots>t_{1}$ be a strictly increasing sequence of positive integers. Let $U_{i}$ be a $t_{i+1}$ by $t_{i}$ matrix of indeterminates over $K, 1 \leq i \leq m$. Let $Y$ be $a 1$ by $r$ matrix of indeterminates, $S$ an $r$ by $r$ symmetric matrix of indeterminates. Then the entries of the 1 by $t_{1}$ product matrix $Y S U_{m} U_{m-1} \cdots U_{1}$ form an $R$-sequence in $K\left[Y, S, U_{i}^{\prime} s\right]$ and the ideal generated is prime.

Proof. By Proposition 4.3, $F=Y S$ has entries which form an $R$-sequence. Proposition 22 of [11] applied to $F U_{m}=Y S U_{m}$ gives the result that the entries of $Y S U_{m}$ form an $R$-sequence and generate a prime ideal. The result for $Y S U_{m} \cdots U_{1}$ then follows by trivial induction on $m$, using Proposition 22 of [11]. 
Proposition 4.7. Let $H=\left(s_{0}, \ldots, s_{m}\right)$ be a standard description with $s_{0}=0$, $s_{m}=s, m<s$. Then if $n=s_{k}<s, J_{H, n}(X)$ is prime.

Proof. If $n=0$, we are done by Proposition 4.2. For $n>0$, let $W=Z^{t} S Z$ be as in Proposition 4.2. Let $Y$ be the first row of $Z^{t}$ (note that $Y$ is the transpose of the first column of $\left.U_{m}\right)$. Then $P=I_{0}\left(Y S U_{m-1} \cdots U_{k}\right)$ is prime in $K\left[Y, U_{m-1}, \ldots U_{k}\right]$ by Proposition 4.6, and so generates a prime in $K\left[U_{i}, V_{i}, S\right]$. Let $\phi^{\prime}: K[X] \rightarrow K\left[U_{i}, V_{i}, S\right] / P$ be the result of composing $\phi$ ( $\phi$ as in Proposition 4.2) with the natural homomorphism whose kernel is $P$. We need only show that $\operatorname{ker} \phi^{\prime}=J_{H, n}$, since $K\left[U_{i}, V_{i}, S\right] / P$ is a domain.

The last $s_{k}$ entries of the first row of $W$ are given by

$$
\begin{aligned}
& Y S\left[\left(U_{m-1} \cdots U_{k} V_{k}\right) * \cdots *\left(U_{m-1} \cdots U_{1} V_{1}\right)\right] \\
& \quad=Y S U_{m-1} \cdots U_{k}\left(V_{k} * \cdots * U_{k-1} \cdots U_{1} V_{1}\right),
\end{aligned}
$$

so $I_{H, n}(X) \subset \operatorname{ker} \phi^{\prime} \Rightarrow J_{H, n}(X) \subset \operatorname{ker} \phi^{\prime}$.

To complete the proof, we need $\operatorname{ker} \phi^{\prime} \subset J_{H, n}$, which will follow if we can show, for each $Q$ prime in $K[X]$ such that $I_{H, n} \subset Q$, ker $\phi^{\prime} \subset Q$.

As in Proposition 4.2, consider $X^{-}$the image of $X$ in $D=K[X] / Q$, and let $L$ be the fraction field of $D$. We need to show that $X^{-}$can be "factored" in the same form as $W$ over $L$ so that $Y^{\prime} S^{\prime} U_{m-1}^{\prime} \cdots U_{k}^{\prime}=0$, where $Y^{\prime}$ is the first row of $Z^{\prime \prime}$.

To do this proceed as in Proposition 4.2, noting that since the column space of $T_{k}$ equals the column space of $Y_{k} * \cdots * Y_{1}$, its first row consists entirely of zeros. But $T_{k}=Z^{\prime t} S^{\prime} U_{m-1}^{\prime} \cdots U_{k}^{\prime}$, so its first row is $Y^{\prime} S^{\prime} U_{m-1}^{\prime} \cdots U_{k}^{\prime}$. Q.E.D.

5. Proof that the ideals $I_{H, n}(X)$ are radical. In this section we prove that the ideals $I_{H, n}(X)$ are radical when $X$ consists of indeterminates.

Proposition 5.1. Let $H=\left(s_{0}, \ldots, s_{m}\right)$ be a standard description, $m<s$. If $s_{h}<n<s_{h+1}$, let $H^{\prime}=\left(s_{0}, \ldots, s_{h-1}, n, s_{h+1}, \ldots, s_{m}\right)$ and $n^{\prime}=s_{h+1}$. Then $J_{H, n}$ $=J_{H^{\prime}, n} \cap J_{H, n^{\prime}}$.

Proof. Since $J_{H, n} \subset J_{H^{\prime}, n}$ and $J_{H, n} \subset J_{H, n^{\prime}}$, we need only show that $J_{H^{\prime}, n} \cap J_{H, n^{\prime}}$ $\subset J_{H, n}$, which will follow from $I_{H^{\prime}, n} \cdot I_{H, n^{\prime}} \subset I_{H, n^{*}}$ Note that $I_{H^{\prime}, n}=I_{H, n}+I_{h}\left(\left.X\right|_{n}\right)$. Also, $I_{H, n^{\prime}}=I_{H, n}+\left(x_{1, s-n}, \ldots, x_{1, s-n^{\prime}+1}\right)$. Hence it is sufficient to show that if $s-n^{\prime}+1 \leq k \leq s-n$ and $M$ is any $h+1$ by $h+1$ minor of $\left.X\right|_{n}$, then $x_{k} M$ is in $I_{H, n}$.

If the first row of $M$ is in the first row of $X$, the result is clear, since then $M \in\left(x_{1, s}, \ldots, x_{1, s-n+1}\right) \subset I_{H, n^{*}}$ Otherwise, let $M^{*}$ be the unique $h+2$ by $h+2$ minor of $X$ which contains $M$ and $x_{k} . M^{*} \in I_{H, n}$, since $k \geq s-n^{\prime}+1$. The expansion of $M^{*}$ with respect to its first row contains $\pm x_{k} M$ and other terms in $\left(x_{1, s-n+1}, \ldots, x_{1, s}\right)$, so $x_{k} M \in I_{H, n}$.

Note that $H^{\prime}$ will not have initial term zero if $s_{0}<n<s_{1}$ and will not have final term $s$ if $s_{m-1}<n<s_{m}$. 
Proposition 5.2. Let $X$ be an $s$ by symmetric matrix of indeterminates over $K, a$ Noetherian domain. Let $H=\left(s_{0}, \ldots, s_{m}\right)$ be a standard description with $s_{m}=s$. Let $n$ be an integer, $0 \leq n \leq s$. Then $I_{H, n}$ is radical.

Proof. By induction, we may assume the result for smaller symmetric matrices. The result is trivial for a 1 by 1 matrix. By Noetherian induction, assume $I_{H, n}(X)$ is a maximal member of the set of ideals for which the conclusion does not hold. Hence any ideal of the class properly containing $I_{H, n}(X)$ is radical. Suppose $s_{0}>0$. Then $K[X] / I_{H, n}(X) \cong K\left[X^{\prime}\right] / I_{H^{\prime}, n^{\prime}}\left(X^{\prime}\right)$, where $X^{\prime}$ is the $s-s_{0}$ by $s-s_{0}$ symmetric matrix of indeterminates obtained by deleting the last $s_{0}$ rows and columns of $X, H^{\prime}=\left(s_{0}-s_{0}, s_{1}-s_{0}, \ldots, s_{m}-s_{0}\right)$ and $n^{\prime}=\max \left\{0, n-s_{0}\right\}$. Since $I_{H^{\prime}, n^{\prime}}\left(X^{\prime}\right)$ is assumed radical by the inductive hypothesis, $K\left[X^{\prime}\right] / I_{H^{\prime}, n^{\prime}}\left(X^{\prime}\right)$ is a reduced ring (i.e. has no nontrivial nilpotents), so $I_{H, n}(X)$ is radical. Hence we may assume $s_{0}=0$.

Suppose $n=s$. Then $K[X] / I_{H, n}(X) \cong K\left[X^{\prime}\right] / I_{H, 0}\left(X^{\prime}\right)$ where $X^{\prime}$ is the $s-1$ by $s-1$ symmetric matrix obtained by deleting the first row and column of $X$. Since $I_{H, 0}\left(X^{\prime}\right)$ is assumed radical, $I_{H, n}(X)$ is radical. Hence we may assume $n<s$.

We use Proposition 24 of [11] to complete the proof. Let $x=x_{1, s-n}$. We show that either condition 3) or condition 4) of [11, Proposition 24] holds. If $n=s_{h}$, $J_{H, n}$ is prime by Proposition 4.7, and $I_{H, n}(X)+(x)=I_{H, n+1}(X)$, so condition 3) of [11, Proposition 24] holds.

If $s_{h}<n<s_{n+1}, \quad J_{H, n}=J_{H^{\prime}, n} \cap J_{H, n^{\prime}}$ by Proposition 5.1. $I_{H, n}(X)+(x)$ $=I_{H, n+1}(X)$, so to show that condition 4) of [11, Proposition 24] holds, we need to show that $I_{H^{\prime}, n} \subset I_{H, n}:(x)$ and $J_{H, n}:(x) \subset J_{H^{\prime}, n}$. The first is equivalent to $x I_{H^{\prime}, n} \subset I_{H, n}$, which was shown in the proof of Proposition 5.1. The second follows from

$$
\begin{aligned}
J_{H, n}:(x) & =\left[J_{H^{\prime}, n} \cap J_{H, n^{\prime}}\right]:(x) \\
& =\left[J_{H^{\prime}, n}:(x)\right] \cap\left[J_{H, n^{\prime}}:(x)\right]=J_{H^{\prime}, n}:(x)
\end{aligned}
$$

since $x \in J_{H, n^{\prime}}$. But $J_{H^{\prime}, n}:(x)=J_{H^{\prime}, n}$ since $J_{H^{\prime}, n}$ is prime by Proposition 4.7. Q.E.D.

The results of $\S \S 4$ and 5 are summarized in

Proposition 5.3. With the same hypotheses as Propositions 5.1 and 5.2, we have:

(1) If $n=s_{h}, I_{H, n}(X)$ is prime.

(2) If $s_{h}<n<s_{h+1}, I_{H, n}(X)=I_{H^{\prime}, n}(X) \cap I_{H, n^{\prime}}(X)$ is the decomposition of the radical ideal $I_{H, n}(X)$ as an intersection of prime ideals.

6. The grade of $I_{H, n}(X)$. To compute gr $I_{H, n}(X)$ we first consider the case where $K$ is a field and $I_{H, n}(X)$ is a prime ideal of $K[X]$.

Proposition 6.1. Let $K$ be a field, $H$ a standard description, $H=\left(s_{0}, \ldots, s_{m}\right)$, $n=s_{h}<s$. Then $\operatorname{dim} I_{H, n}=s m-h-s_{1}-\cdots-s_{m-1}$. 
Proof. Recall that $\operatorname{dim} I_{H, n}=\operatorname{dim} K[X] / I_{H, n}$. To obtain the result we construct a new generic point for $I_{H, n}$ onto a ring that has the required dimension.

To simplify notation, let $w_{i}=s-i+1,1 \leq i \leq s$. Given $H$ and new indeterminates $u_{i j}$, we construct a symmetric $s$ by $s$ matrix $U$ over $K\left(u_{i j}\right)$ that has the same vanishing of subdeterminants as required by $H$. Fill in the last column of $U$ with $s-1$ indeterminates $u_{2, w_{1}}, u_{3, w_{1}}, \ldots, u_{s, w_{1}}$ if $n>0$, with 0 as the first entry, and with $s$ indeterminates $u_{1, w_{1}}, u_{2, w_{1}}, \ldots, u_{s, w_{1}}$ if $n=0$. To impose symmetry, the last row of $U$ is also determined. Fill in the remaining rightmost $s_{1}$ columns over $K\left(u_{i, w_{1}}\right)$ so that the 2 by 2 minors vanish. Since the last row was already determined, this uniquely determines the entries of these columns. Now fill in the remaining bottom $s_{1}$ rows of $U$ by symmetry. Assume inductively that we have constructed $U$ so that the rightmost $s_{i}$ columns and bottom $s_{i}$ rows are determined. That is, in Figure 1, we have filled in up to but not including the crosshatched area.

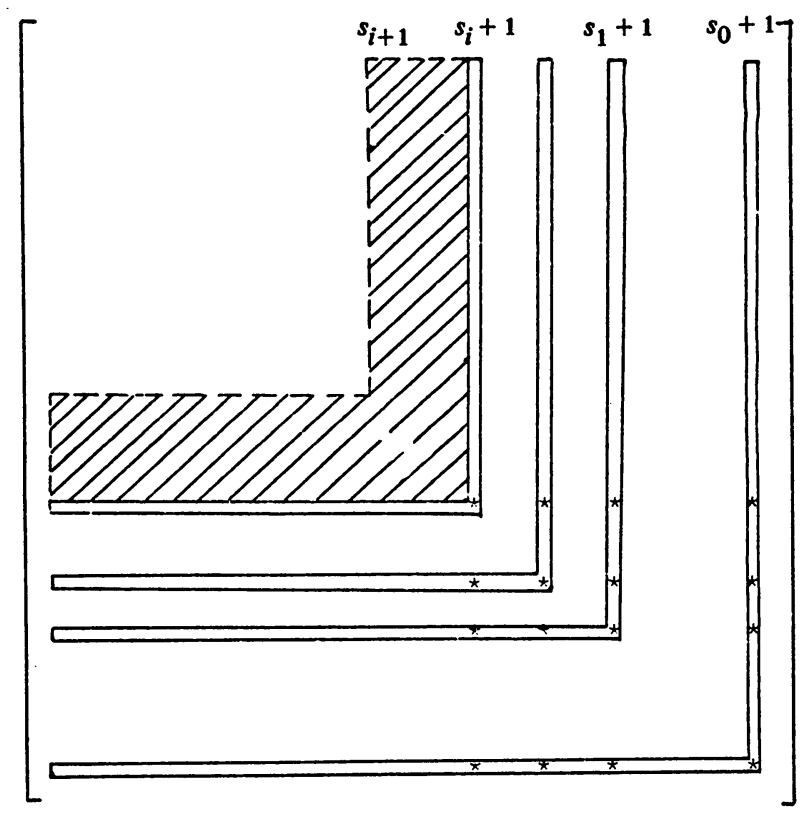

Figure 1

Now fill in the $w_{s_{i}+1}$ th column with indeterminates $u_{1, w_{s}+1}, u_{2, w_{s i}+1}, \ldots, u_{s-s_{i}, w_{s i}+1}$ if $i>h$, and replace $u_{1, w_{s+1}}$ with 0 if $i \leq h$. This completes the $w_{s_{i}+1}$ th column and, by symmetry, the $w_{s_{i}+1}$ th row of $U$. Now the crosshatched block is completely determined. To see this, note that the $i+1$ "arbitrary" columns completed so far intersect the corresponding $i+1$ rows in an invertible matrix $A_{i+1}$ whose entries are distinguished by $*$ in Figure 1 . (The matrix is invertible since it is a symmetric matrix of distinct (except for symmetry) indeterminates.) The $w_{s_{i}+2}$ th column intersected with the rows of $A_{i+1}$ is uniquely expressible as a linear combination 
of the $i+1$ columns of $A_{i+1}$. But the entire $w_{s_{i}+2}$ th column must be the same combination of the $i+1$ columns that form $A_{i+1}$; define the entries in that way. Fill in the $w_{s_{i}+2}$ th row by symmetry. We can similarly fill in the entire crosshatched block. At each step it is clear that we obtain a symmetric matrix satisfying the conditions imposed by $H$ and $n$.

Let $\phi: K[X] \rightarrow K[U] \subset K\left(u_{i j}\right)$ take $X$ to $U$. I claim that $\operatorname{ker} \phi=I_{H, n}(X)$. It is clear that $I_{H, n} \subset \operatorname{ker} \phi$. Since $I_{H, n}$ is prime, we need only show that $X^{-}$"factors" in the same form as $U$ over $L$, the fraction field of $D=K[X] / I_{H, n}$. First note that the $w_{s_{i}+1}$ columns of $X^{-}$are linearly independent, $0 \leq i<m$. This follows by specializing the entries of $X$ by specializing the $x_{s-i, w_{s+1}}$ entries to 1 and all other entries to 0 . Then $I_{H, n}(X)$ specializes to (0) while the $w_{s_{i}+1}$ columns specialize to distinct columns of the $s$ by $s$ identity matrix. It follows that in a homomorphic image of $D$, the $w_{s_{i}+1}$ columns are independent, and so are independent in $D$.

Since the rank of the last $s_{i}$ columns is at most $i$, and we have $i$ independent columns, all of the other columns are linearly dependent on them. As with $U$, the symmetry uniquely determines the linear combination, so $X^{-}$"factors" in the same form as $U$.

Hence $\operatorname{dim} I_{H, n}=\operatorname{dim} K[U]$. But $K\left[u_{i j}\right] \subset K[U] \subset K\left(u_{i j}\right)$, so $\operatorname{dim} K[U]$ $=\operatorname{tr} \operatorname{deg}_{K} K[U]$, which is simply the number of indeterminates $u_{i j}$, which is $(s-1)+\left(s-s_{1}-1\right)+\cdots+\left(s-s_{h-1}-1\right)+\left(s-s_{h}\right)+\cdots+\left(s-s_{m-1}\right)$ $=s m-h-s_{1}-\cdots-s_{m-1}$. Q.E.D.

Proposition 6.2. For any Noetherian domain $K$, if $H$ is a standard description, $n=s_{h}<s$, then gr $I_{H, n}=g_{H, n}$ where $g_{H, n}$ is defined as $s(s+1) / 2-s m+h+s_{1}$ $+\cdots+s_{m-1}$.

Proof. If $K$ is Cohen-Macaulay, grade and rank are equal in $K[X]$, and the rank of $I_{H, n}$ is unaffected by localizing at $K-\{0\}$. Hence the grade of $L_{H, n}$ in $K[X]$ is the same as the rank of $I_{H, n}$ in $L[X]$, where $L$ is the fraction field of $K$. But, in $L[X], \quad \operatorname{rank} I_{H, n}=\operatorname{dim} L[X]-\operatorname{dim} I_{H, n}=s(s+1) / 2-\operatorname{dim} I_{H, n}=g_{H, n} \quad$ by Proposition 6.1.

We obtain the result for arbitrary Noetherian domains $K$ by utilizing results of Hochster. When $K=Z$, the integers, we have gr $I_{H, n}=g_{H, n}$ since $Z$ is CohenMacaulay. $I_{H, n}$ is prime and $I_{H, n} \cap Z=(0)$; hence $Z[X] / I_{H, n}$ is $Z$-flat. By Theorem 2 of [9], $I_{H, n}$ is grade stable, implying that for any Noetherian domain $K$, gr $I_{H, n}$ in $K[X]$ is at least $g_{H, n}$. Localizing at $K-\{0\}$ cannot decrease the grade, but after localization the grade is $g_{H, n}$. Q.E.D.

We now consider the case where $I_{H, n}$ is not prime. The desired result holds only if $n=s_{h}+1$.

Proposition 6.3. For any Noetherian domain $K$, let $H$ be a standard description, $n=s_{h}+1<s_{h+1}$. Let $H^{\prime}, n^{\prime}$ be as in Proposition 5.1. Then gr $I_{H, n}=\operatorname{gr} I_{H^{\prime}, n}$ $=\operatorname{gr} I_{H, n^{\prime}}$

Proof. This follows easily from the grade formula of Proposition 6.2. 
Proposition 6.4. With the same hypothesis as Proposition 6.3. $I_{H^{\prime}, n}+I_{H, n^{\prime}}=I_{H^{\prime}, n^{\prime}}$ and $\mathrm{gr} I_{H^{\prime}, n^{\prime}}=\operatorname{gr} I_{H, n}+1$.

Proof. The first assertion is obvious; the second follows from the formula for gr $I_{H^{\prime}, n^{\prime}}$

7. Completion of the proof of Theorem 1. Let $K$ be a Noetherian domain; let $X$ be an $s$ by $s$ symmetric matrix of indeterminates. We need to show that the ideals $I_{H, n}(X)$ are perfect when $n=s_{h}$ or $n=s_{h}+1$. By Proposition 5.3, when $K=Z$, the integers, no integer is a zero-divisor on $I_{H, n}$, so that $Z[X] / I_{H, n}$ is faithfully $Z$ flat. By Proposition 20 of [11] the result will follow for all $K$ if we can obtain the result when $K$ is a finite prime field; hence we may assume $K$ is a field.

Given $I_{H, n}(X)$, assume inductively that the result is proved for smaller matrices and larger ideals of the form $I_{H^{\prime}, n^{\prime}}(X)$, where $H$ is a standard description and $n=s_{h}$ or $n=s_{h+1}$. As in the proof of Proposition 5.2, we may reduce to the case where $n<s$.

If $n=s_{h}, I_{H, n}$ is prime by Proposition 5.3(1). Further, $I_{H, n}$ is homogeneous and $x=x_{1, s-n+1}$ is a form not a zero-divisor on $I_{H, n}$. But $I_{H, n}+(x)=I_{H, n+1}$ and $I_{H, n}$ are perfect or not alike, by the corollary to [11, Proposition 19]. $I_{H, n+1}$ is perfect by induction, so we are done if $n=s_{h}$.

If $n=s_{h}+1$, we have $I_{H, n}=I_{H^{\prime}, n} \cap I_{H, n^{\prime}}$ by Proposition 5.3(2). By induction, $I_{H^{\prime}, n}, I_{H, n^{\prime}}$, and $I_{H^{\prime}, n^{\prime}}=I_{H^{\prime}, n}+I_{H, n^{\prime}}$ are all perfect. By Proposition 6.3 and Proposition 6.4, the grade requirements of Proposition 18 of [11] are fulfilled, and hence $I_{H, n}$ is perfect by [11, Proposition 18].

8. $K[X] / I_{H}(X)$ as a ring of invariants. In this section we complete the results introduced in $\S 2$. Throughout this section, $K$ is a Noetherian domain and $X$ is an $s$ by $s$ symmetric matrix of indeterminates over $K$.

Proposition 8.1. Using the notation of Proposition 4.2, $K\left[Z^{t} Z\right] \cong K\left[Z^{t} S Z\right]$ under the mapping that takes $Z^{t} Z$ to $Z^{t} S Z$.

Proof. Let $\phi: K[X] \rightarrow K\left[Z^{t} S Z\right]$ be as in Proposition 4.2, so $\operatorname{ker} \phi=I_{H}(X)$. Define $\pi: K[X] \rightarrow K\left[Z^{t} Z\right]$ taking $X$ to $Z^{t} Z$. It suffices to show $\operatorname{ker} \pi=I_{H}(X)$. Clearly, $I_{H}(X) \subset$ ker $\pi$. Let $T$ be a $t_{m}$ by $t_{m}$ matrix of new indeterminates, and let $\alpha: K\left[Z^{t} S Z\right] \rightarrow K\left[Z^{t} T^{t} T Z\right]$ take $Z^{t} S Z$ to $Z^{t} T^{t} T Z$. Then $\alpha$ is an isomorphism. It is clearly onto, but it is induced by $\alpha^{\prime}: K[Z, S] \rightarrow K\left[Z, T^{t} T\right]$ where $\alpha^{\prime}$ takes $Z$ to $Z$ and $S$ to $T^{t} T$. To see that this is one-to-one, it suffices to show that the entries of $T^{t} T$ are algebraically independent over $L$, the algebraic closure of the fraction field of $K$. For this it suffices to show that a Zariski dense subset of the symmetric matrices over $L$ can be factored $B^{t} B$. Consider the matrices with all distinct eigenvalues. We show each such matrix can be so factored. Let $M$ be given, $M$ symmetric with distinct eigenvalues. Then $M=A D A^{-1}$ where $D$ is diagonal and $A$ is a change of basis matrix taking the standard basis to an eigenvector basis. Hence $A$ is uniquely determined up to nonzero multiples of its 
columns. But $M^{t}=M=\left(A^{-1}\right)^{t} D^{t} A^{t}$, so $\left(A^{-1}\right)^{t}$ has columns which are nonzero multiples of the columns of $A$. Hence $\left(A^{-1}\right)^{t}=A C, C$ diagonal. Thus $A^{-1}$ $=\left(A^{-1}\right)^{t}=\left(\left(A^{-1}\right)^{t}\right)^{t}=(A C)^{t}=C^{t} A^{t}=C A^{t}$, so $M=A D A^{-1}=A D C A^{-1}$ $=B B^{t}$, where $B=A E$ and $E=\left(e_{i j}\right)$, where $e_{i i}=\sqrt{ } d_{i} c_{i}$ and $e_{i j}=0, i \neq j$, as required.

Hence we have the following diagram:

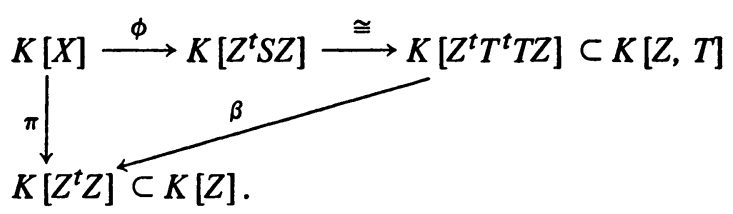

Since $I_{H}=\operatorname{ker} \phi \subset \operatorname{ker} \pi$, we have the $\operatorname{map} \beta: K\left[Z^{t} T^{t} T Z\right] \rightarrow K\left[Z^{t} Z\right]$. To complete the proof, note that the one-to-one map $\beta^{*}: K[Z] \rightarrow K[Z, T]$ that takes $Z$ to $T Z$ induces $\beta^{-1}: K\left[Z^{t} Z\right] \rightarrow K\left[Z^{t} T^{t} T Z\right]$, where $\beta^{-1}$ takes $Z^{t} Z$ to $Z^{t} T^{t} T Z$, so $\beta$ is an isomorphism. Hence $\operatorname{ker} \pi=\operatorname{ker} \phi=I_{H}(X)$. Q.E.D.

We will be dealing with matrices of the same form as $W$ in Proposition 4.2, and introduce some conventions convenient for this purpose. If $U_{m-1}, \ldots, U_{0}$ and $V_{m}, \ldots, V_{0}$ are sequences of matrices such that the products $U_{k} V_{k}, 0 \leq k$ $\leq m-1$, and $U_{m-1} \cdots U_{0}$ are all defined, and $V_{m}$ and $U_{m-1}$ have the same number of rows, write $U * V$ for $V_{m} *\left(U_{m-1} V_{m-1}\right) * \cdots *\left(U_{m-1} \cdots U_{0} V_{0}\right)$.

If a matrix of the form $Z=U * V$ arises in the construction of a generic point for an ideal $I_{H}(X)$ as in Proposition 4.2, then

(1) $U_{m-1}$ has $t_{m}<s$ rows, and $U_{k-1}$ has strictly less rows than $U_{k}, 1 \leq k$ $\leq m-1$.

(2) $V_{k}$ has at least one column unless $k=0$, and the sum of the number of columns of the $V_{k}$ 's is $s$.

Conversely, if $Z=U * V$ and the dimensions of the $U$ 's and $V$ 's satisfy (1) and (2), then there exists $H$ such that $K[X] / I_{H} \cong K\left[Z^{\prime} Z\right]$. This $H$ is found by: $j_{k}=$ the number of columns of $V_{k}, i_{k}=j_{k}+i_{k-1}$ where $i_{-1}=0$, and $t_{k}=$ the number of rows of $V_{k}$. We say that $Z=U * V$ has form $H$ in this case. In any case, $m$ is the length of $U * V$. We say that $U * V$ is composed of indeterminates if all entries of the component matrices $U_{k}, V_{k}$, considered together, are algebraically independent over $K$.

Proposition 8.2. Let $Z=U * V$ be $t$ by $s, t<s$, where $Z$ is composed of indeterminates. Let $\phi: K[X] \rightarrow K\left[Z^{t} Z\right]$ take $X$ to $Z^{\prime} Z$. Then $\operatorname{ker} \phi=I_{H}(X)$ for some $H$. ( $H$ will not, in general, be a standard description.)

Proof. If $Z$ is of the form $H$, we are done. We proceed by induction on $m$. If $m=1$, we are done by $[16, \S 14]$. Assume the result for $m^{\prime}<m$, where $m \geq 2$. $Z=V_{m} * U_{m-1}\left[V_{m-1} * \cdots *\left(U_{m-2} \cdots U_{0} V_{0}\right)\right]=V_{m} * U_{m-1} Z^{\prime}$ where $Z^{\prime}$ is of length $m-1$. Hence $Z^{\prime}$ is of the form $H^{\prime}$ by induction, where $H^{\prime}=\left\{\left(t_{0}, i_{0}\right), \ldots\right.$, $\left.\left(t_{m-1}, i_{m-1}\right)\right\} . V_{m}$ is $t$ by $r$, and $U_{m-1}$ is $t$ by $i_{m-1}$. If $t>t_{m-1}$, then $Z$ is of form $H$, 
where $t_{m}=t, i_{m}=i_{m-1}+r$, and $H=H^{\prime} \cup\left\{\left(t_{m}, i_{m}\right)\right\}$. If $t \leq t_{m}$, rewrite $Z$ by replacing $V_{m} * U_{m-1} V_{m-1}$ by $V_{m-1}^{\prime}$ and $U_{m-1} U_{m-2}$ by $U_{m-2}^{\prime}$. These are still composed of indeterminates (this follows from $[16, \S 14]$ ), and the result follows by induction. Q.E.D.

We next show that if $Z=U * V$ is composed of indeterminates and $K$ is a field of characteristic zero, then $K\left[Z^{t} Z\right]$ is the ring of invariants of a rational representation of a product of an orthogonal group and a product of general linear groups on $K[U, V]$. Let $U_{m-1}, \ldots, U_{0}$ and $V_{m}, \ldots, V_{0}$ be such that $U_{k}$ is $t_{k+1}$ by $t_{k}, V_{k}$ is $t_{k}$ by $j_{k}$. Let $G=O\left(t_{m}, K\right) \times\left(\prod_{k=0}^{m-1} \mathrm{GL}\left(t_{k}, K\right)\right) . G$ is a reductive linear algebraic group. Let a matrix $A \in O\left(t_{m}, K\right)$ act on the 1-forms of $K[U, V]$ by taking $V_{m}$ onto $A V_{m}$ and $U_{m-1}$ onto $A U_{m-1}$. Let $B \in \mathrm{GL}\left(t_{k}, K\right)$ act on the 1forms of $K[U, V]$ by taking $U_{k}$ onto $U_{k} B^{-1}$ and $U_{k-1}$ onto $B U_{k-1}$. Let $V_{k}$ go onto $B V_{k} . A$ and $B$ leave all other $U$ 's and $V$ 's fixed. Clearly these actions of the various components of $G$ commute with one another, so that they define an action of $G$ on the 1 -forms of $K[U, V]$ which extends to an action of $G$ on $K[U, V]$.

Proposition 8.3. The ring of invariants of the above action of $G$ on $K[U, V]$ is $K\left[Z^{\imath} Z\right]$. Hence, for a suitable matrix of indeterminates $X$ and description $H$, $K[U, V]^{G} \cong K[X] / I_{H}(X)$.

Proof. It follows easily from [11, Proposition 28], that the ring of invariants of $\Pi \underset{k=0}{m-1} \mathrm{GL}\left(t_{k}, K\right)$ is $R^{\prime}=K[Z] . O\left(t_{m}, K\right)$ acts on $R^{\prime}$ by taking $Z$ to $A Z$ for $A \in O\left(t_{m}, K\right)$. The ring of invariants of this action is known classically [16, §17] to be $K\left[Z^{\prime} Z\right]$ when $Z$ consists of indeterminates. Let $Z^{\prime}$ be a matrix of indeterminates the same size as $Z$, and let $\phi: K\left[Z^{\prime}\right] \rightarrow K[Z]$ take $Z^{\prime}$ to $Z$. Let $G^{\prime}=O\left(t_{m}, K\right)$ act on $Z^{\prime}$ in the same manner as it acts on $Z$. Then $K\left[Z^{\prime}\right]^{G^{\prime}}$ $=K\left[Z^{\prime \prime} Z^{\prime}\right]$. By $\left[8\right.$, p. 156], since $G$ is reductive, $K[Z]^{G}=K\left[Z^{\prime} Z\right]$. Q.E.D.

Corollary. If $K$ has characteristic 0 , the ring of invariants of Proposition 8.3 is Cohen-Macaulay.

The ring is Cohen-Macaulay regardless of the characteristic of $K$; we do not know whether it is a ring of invariants.

\section{BIBLIOGRAPHY}

1. M. Auslander and D. A. Buchsbaum, Codimension and multiplicity, Ann. of Math. 68 (1958), 625-657. MR 20 \#6414.

2. A. Borel, Linear algebraic groups, Benjamin, New York, 1969. MR 40 \#4273.

3. D. A. Buchsbaum and D. S. Rim, A generalized Koszul complex. II. Depth and multiplicity, Trans. Amer. Math. Soc. 111 (1964), 197-224. MR 28 \#3076.

4. W. L. Chow, On unmixedness theorem, Amer. J. Math. 86 (1964), 799-822. MR 30 \#2031.

5. J. A. Eagon, Ideals generated by the subdeterminants of a matrix, Thesis, University of Chicago, Chicago, Ill., 1961.

6. J. A. Eagon and D. G. Northcott, Ideals defined by matrices and a certain complex associated with them, Proc. Roy. Soc. Ser. A 269 (1962), 188-204. MR 26 \#161. 
7. J. A. Eagon and D. G. Northcott, Generically acyclic complexes and generically perfect ideals, Proc. Roy. Soc. Ser. A 299 (1967), 147-172. MR 35 \#5435

8. J. Fogarty, Invariant theory, Benjamin, New York, 1969. MR 39 \# 1458.

9. M. Hochster, Generically perfect modules are strongly generically perfect, Proc. London Math. Soc. (3) 23 (1971), 477-488.

10. - Rings of invariants of tori, Cohen-Macaulay rings generated by monomials, and polytopes 96 (1972), 318-337.

11. M. Hochster and J. A. Eagon, Cohen-Macaulay rings, invariant theory, and the generic perfection of determinantal loci, Amer. J. Math. 93 (1971), 1020-1058.

12. I. Kaplansky, Commutative rings, Allyn and Bacon, Boston, Mass., 1970. MR 40 \#234.

13. F. S. Macaulay, The algebraic theory of modular systems, Cambridge Tracts 19 (1916).

14. M. Nagata, Local rings, Interscience Tracts in Pure and Appl. Math., no. 13, Interscience, New York, 1962. MR 27 \#5790.

15. D. W. Sharpe, On certain polynomial ideals, defined by matrices, Quart. J. Math. Oxford Ser. (2) 15 (1964), 155-175. MR 29 \#1226.

16. H. Weyl, The classical groups. Their invariants and representations, 2 nd ed., Princeton Univ. Press, Princeton, N. J., 1946.

17. O. Zariski and P. Samuel, Commutative algebra. Vols. I, II, University Series in Higher Math., Van Nostrand, Princeton, N. J., 1958, 1960. MR 19, 833; 22 \#11006.

School of Mathematics, University of Minnesota, Minneapolis, Minnesota 55455

Current address: Center for Teaching and Learning, University of North Dakota, Grand Forks, North Dakota 58201 